\title{
Certain Integral Transform and Fractional Integral Formulas for the Generalized Gauss Hypergeometric Functions
}

\author{
Junesang Choi ${ }^{1}$ and Praveen Agarwal ${ }^{2}$ \\ ${ }^{1}$ Department of Mathematics, Dongguk University, Gyeongju 780-714, Republic of Korea \\ ${ }^{2}$ Department of Mathematics, Anand International College of Engineering, Jaipur, Near Kanota, Agra Road, Bassi, Jaipur 303012, India
}

Correspondence should be addressed to Junesang Choi; junesang@mail.dongguk.ac.kr

Received 25 April 2014; Revised 23 May 2014; Accepted 4 June 2014; Published 16 June 2014

Academic Editor: Soheil Salahshour

Copyright (c) $2014 \mathrm{~J}$. Choi and P. Agarwal. This is an open access article distributed under the Creative Commons Attribution License, which permits unrestricted use, distribution, and reproduction in any medium, provided the original work is properly cited.

\begin{abstract}
A remarkably large number of integral transforms and fractional integral formulas involving various special functions have been investigated by many authors. Very recently, Agarwal gave some integral transforms and fractional integral formulas involving the $F_{p}^{(\alpha, \beta)}(\cdot)$. In this sequel, using the same technique, we establish certain integral transforms and fractional integral formulas for the generalized Gauss hypergeometric functions $F_{p}^{(\alpha, \beta, m)}(\cdot)$. Some interesting special cases of our main results are also considered.
\end{abstract}

\section{Introduction and Preliminaries}

The theory of special functions has been one of the most rapidly growing research subjects in mathematical analysis. A lot of special functions of mathematical physics and engineering, such as Jacobi and Laguerre polynomials, can be expressed in terms of the generalized hypergeometric functions or confluent hypergeometric functions (see, e.g., [1, pages 66-69]). Therefore, the corresponding extensions of several other familiar special functions are expected to be useful and need to be investigated (see, e.g., [2-7] and, for a very recent work, see also [8]).

Very recently, Parmar [9] introduced and investigated some fundamental properties and characteristics of the more generalized beta type function $B_{p}^{(\alpha, \beta ; m)}(x, y)$ defined by (see [9, page 37, Equation (19)])

$$
\begin{gathered}
B_{p}^{(\alpha, \beta ; m)}(x, y):=\int_{0}^{1} t^{x-1}(1-t)^{y-1}{ }_{1} F_{1}\left(\alpha ; \beta ; \frac{-p}{t^{m}(1-t)^{m}}\right) d t \\
(\mathfrak{R}(p)>0 ; \min \{\Re(x), \mathfrak{R}(y), \mathfrak{R}(\alpha), \mathfrak{R}(\beta)\} 0, \\
\mathfrak{R}(m)>0) .
\end{gathered}
$$

It is easy to see that the special case of (1) when $m=1$ reduces to the well-known generalized beta type function defined by (see, e.g., [7, page 4602, Equation (4)]; see also [6, page 32, Chapter 4])

$$
\begin{aligned}
& B_{p}^{(\alpha, \beta)}(x, y)=\left(B_{p}^{(\alpha, \beta ; 1)}(x, y)\right) \\
&=\int_{0}^{1} t^{x-1}(1-t)^{y-1}{ }_{1} F_{1}\left(\alpha ; \beta ; \frac{-p}{t(1-t)}\right) d t \\
&(\Re(p)>0 ; \min \{\Re(x), \mathfrak{R}(y), \mathfrak{R}(\alpha), \mathfrak{R}(\beta)\}>0) .
\end{aligned}
$$

For $\alpha=\beta$, (2) reduces to the extended beta type function, due to Chaudhry et al. [4, page 591, Equation (1.7)] (see also [3, page 20, Equation (1.7)]), defined by

$$
\begin{aligned}
B_{p}(x, y) & =\left(B_{p}^{(\alpha, \alpha)}(x, y)\right) \\
& =\int_{0}^{1} t^{x-1}(1-t)^{y-1} \exp \left(\frac{-p}{t(1-t)}\right) d t
\end{aligned}
$$


The classical Euler's beta function $B(x, y)$ is defined by (see, e.g., [1, pages 7-11])

$$
B(x, y):=\int_{0}^{1} t^{x-1}(1-t)^{y-1} d t \quad(\mathfrak{R}(x)>0, \mathfrak{R}(y)>0) .
$$

It is easy to see the following relation:

$$
B(x, y):=B_{0}(x, y)=B_{0}^{(\alpha, \beta)}(x, y)=B_{0}^{(\alpha, \beta ; 1)}(x, y) .
$$

By making use of $B_{p}(x, y)$, Chaudhry et al. [4, page 591, Equations (2.1) and (2.2)] extended the Gauss's hypergeometric function as follows:

$$
F_{p}(a, b ; c ; z):=\sum_{n=0}^{\infty}(a)_{n} \frac{B_{p}(b+n, c-b)}{B(b, c-b)} \frac{z^{n}}{n !} \quad(|z|<1),
$$

where $\mathfrak{R}(c)>\mathfrak{R}(b)>0$ and $\mathfrak{R}(p) \geq 0$ and $(a)_{n}$ denotes the Pochhammer symbol given in (11).

Similarly, by appealing to $B_{p}^{(\alpha, \alpha)}(x, y)$, Özergin et al. introduced and investigated a further extension of the following potentially useful generalized Gauss hypergeometric functions defined as follows (see, e.g., [7, page 4606, Section 3]; see also [6, page 39, Chapter 4]):

$$
F_{p}^{(\alpha, \beta)}(a, b ; c ; z)=\sum_{n=0}^{\infty}(a)_{n} \frac{B_{p}^{(\alpha, \beta)}(b+n, c-b)}{B(b, c-b)} \frac{z^{n}}{n !} \quad(|z|<1),
$$

where $\min \{\mathfrak{R}(\alpha), \mathfrak{R}(\beta)\}>0, \mathfrak{R}(c)>\mathfrak{R}(b)>0$, and $\mathfrak{R}(p) \geq$ 0 .

By using the more generalized beta function (1), Parmar [9] introduced and investigated a family of the following potentially useful generalized Gauss hypergeometric functions defined as follows (see [9, page 44]):

$$
\begin{array}{r}
F_{p}^{(\alpha, \beta ; m)}(a, b ; c ; z)=\sum_{n=0}^{\infty}(a)_{n} \frac{B_{p}^{(\alpha, \beta ; m)}(b+n, c-b)}{B(b, c-b)} \frac{z^{n}}{n !} \\
(|z|<1),
\end{array}
$$

where $\min \{\mathfrak{R}(\alpha), \mathfrak{R}(\beta), \mathfrak{R}(m)\}>0 ; \mathfrak{R}(c)>\mathfrak{R}(b)>0$ and $\mathfrak{R}(p) \geq 0$.

It is obvious to see that

$$
\begin{gathered}
F_{p}^{(\alpha, \beta ; 1)}(a, b ; c ; z)=F_{p}^{(\alpha, \beta)}(a, b ; c ; z), \\
F_{p}^{(\alpha, \alpha ; 1)}(a, b ; c ; z)=F_{p}(a, b ; c ; z), \\
F_{0}^{(\alpha, \alpha ; 1)}(a, b ; c ; z)={ }_{2} F_{1}(a, b ; c ; z),
\end{gathered}
$$

where the ${ }_{2} F_{1}(\cdot)$ is a special case of the well-known generalized hypergeometric series ${ }_{p} F_{q}(\cdot)$ defined by (see, e.g., [1, Section 1.5]; see also [10])

$$
\begin{aligned}
{ }_{p} F_{q}\left[\begin{array}{c}
\alpha_{1}, \ldots, \alpha_{p} ; \\
\beta_{1}, \ldots, \beta_{q} ;
\end{array}\right] & =\sum_{n=0}^{\infty} \frac{\left(\alpha_{1}\right)_{n} \cdots\left(\alpha_{p}\right)_{n}}{\left(\beta_{1}\right)_{n} \cdots\left(\beta_{q}\right)_{n}} \frac{z^{n}}{n !} \\
& ={ }_{p} F_{q}\left(\alpha_{1}, \ldots, \alpha_{p} ; \beta_{1}, \ldots, \beta_{q} ; z\right),
\end{aligned}
$$

where $(\lambda)_{n}$ is the Pochhammer symbol defined (for $\lambda \in \mathbb{C}$ ) by (see [1, page 2 and pages $4-6]$ )

$$
\begin{aligned}
(\lambda)_{n} & := \begin{cases}1 & (n=0) \\
\lambda(\lambda+1), \ldots,(\lambda+n-1) & (n \in \mathbb{N}:=\{1,2,3, \ldots\})\end{cases} \\
& =\frac{\Gamma(\lambda+n)}{\Gamma(\lambda)} \quad\left(\lambda \in \mathbb{C} \backslash \mathbb{Z}_{0}^{-}\right) .
\end{aligned}
$$

Here, $\Gamma$ is the familiar Gamma function and $\mathbb{C}$ and $\mathbb{Z}_{0}^{-}$ denote sets of complex numbers and nonpositive integers, respectively.

The above-mentioned detailed and systematic investigation was indeed motivated largely by a demonstrated potential for applications of the more generalized Gauss hypergeometric function $F_{p}^{(\alpha, \beta ; m)}$ and their special cases to many diverse areas of mathematical, physical, engineering, and statistical sciences (see, for details, [9] and the references cited therein). Very recently, Agarwal [11] gave some interesting integral transform and fractional integral formulas involving (7). In this sequel, using the same technique, we propose to derive some integral transforms and image formulas for the generalized Gauss hypergeometric function (8) by applying certain integral transforms (like beta transform, Laplace transform, and Whittaker transforms) and general pair of fractional integral operators involving Gauss hypergeometric function ${ }_{2} F_{1}$, which will be introduced in Sections 2 and 3, respectively. We also consider some interesting special cases of our main results.

\section{Integral Transform and the Generalized Gauss Hypergeometric Functions}

In this section, we will prove three theorems, which exhibit the connections between the Euler, Laplace, and Whittaker integral transforms and the generalized Gauss hypergeometric type functions $F_{p}^{(\alpha, \beta ; m)}(a, b ; c ; z)$ defined by (8).

Theorem 1. Suppose that $\mathfrak{R}(p) \geq 0, \min \{\mathfrak{R}(\alpha), \mathfrak{R}(\beta)$, $\mathfrak{R}(m)\}>0, \mathfrak{R}(c)>\mathfrak{R}(b)>0$, and $l \in \mathbb{C}$ are parameters. Then, the following beta transform formula holds:

$$
\begin{aligned}
B & \left\{F_{p}^{(\alpha, \beta ; m)}(l+m, b ; c ; y z): l, m\right\} \\
& =B(l, m) \sum_{n=0}^{\infty}(l)_{n} \frac{B_{p}^{(\alpha, \beta ; m)}(b+n, c-b)}{B(b, c-b)} \frac{y^{n}}{n !} \\
& =B(l, m) F_{p}^{(\alpha, \beta ; m)}(l, b ; c ; y) \quad(|y|<1),
\end{aligned}
$$

where the beta transform of $f(z)$ is defined as (see [12])

$$
B\{f(z): a, b\}=\int_{0}^{1} z^{a-1}(1-z)^{b-1} f(z) d z .
$$

Further, it is assumed that the involved Euler (beta) transforms $F_{p}^{(\alpha, \beta ; m)}$ exist. 
Proof. Using (13) and applying (8) to the Euler (beta) transform of (12), we get

$$
\begin{aligned}
& \int_{0}^{1} z^{l-1}(1-z)^{m-1} F_{p}^{(\alpha, \beta ; m)}(l+m, b ; c ; y z) d z \\
& =\int_{0}^{1} z^{l-1}(1-z)^{m-1} \sum_{n=0}^{\infty}(l+m)_{n} \frac{B_{p}^{(\alpha, \beta ; m)}(b+n, c-b)}{B(b, c-b)} \frac{(y z)^{n}}{n !} d z .
\end{aligned}
$$

By changing the order of integration and summation and using beta integral, we obtain

$$
\begin{aligned}
& \int_{0}^{1} z^{l-1}(1-z)^{m-1} F_{p}^{(\alpha, \beta ; m)}(l+m, b ; c ; y z) d z \\
& =\sum_{n=0}^{\infty}(l+m)_{n} \frac{B_{p}^{(\alpha, \beta ; m)}(b+n, c-b)}{B(b, c-b)} \frac{\Gamma(l+n) \Gamma(m)}{\Gamma(l+m+n)} \frac{(y)^{n}}{n !} \\
& =\frac{\Gamma(l) \Gamma(m)}{\Gamma(l+m)} \sum_{n=0}^{\infty}(l)_{n} \frac{B_{p}^{(\alpha, \beta ; m)}(b+n, c-b)}{B(b, c-b)} \frac{(y)^{n}}{n !},
\end{aligned}
$$

which, upon using (8), yields our desired result (12).

Theorem 2. Suppose that $y \geq 0, \mathfrak{R}(s)>0, \min \{\mathfrak{R}(\alpha), \mathfrak{R}(\beta)$, $\mathfrak{R}(m)\}>0, \mathfrak{R}(c)>\mathfrak{R}(b)>0, \mathfrak{R}(p) \geq 0$, and $|y / s|<1$. Then, the following Laplace transform formula holds:

$$
L\left\{z^{l-1} F_{p}^{(\alpha, \beta ; m)}(a, b ; c ; y z)\right\}=\frac{\Gamma(l)}{s^{l}}{ }_{1} F_{p}^{(\alpha, \beta ; m)}\left(a, l, b ; c ; \frac{y}{s}\right),
$$

where the Laplace transform of $f(z)$ is defined as (see [12])

$$
L\{f(z)\}=\int_{0}^{\infty} e^{-s z} f(z) d z
$$

and assume that both sides of (16) exist.

Proof. Using (17) and applying (8), we get

$$
\begin{aligned}
\int_{0}^{\infty} z^{l-1} e^{-s z} F_{p}^{(\alpha, \beta ; m)}(a, b ; c ; y z) d z \\
\quad=\int_{0}^{\infty} z^{l-1} e^{-s z} \sum_{n=0}^{\infty}(a)_{n} \frac{B_{p}^{(\alpha, \beta ; m)}(b+n, c-b)}{B(b, c-b)} \frac{(y z)^{n}}{n !} d z .
\end{aligned}
$$

By changing the order of integration and summation and using Laplace transform, we get

$$
\begin{aligned}
\int_{0}^{\infty} z^{l-1} e^{-s z} F_{p}^{(\alpha, \beta ; m)}(a, b ; c ; y z) d z \\
=\sum_{n=0}^{\infty}(a)_{n} \frac{B_{p}^{(\alpha, \beta ; m)}(b+n, c-b)}{B(b, c-b)} \frac{\Gamma(l+n)}{s^{l+n}} \frac{(y)^{n}}{n !},
\end{aligned}
$$

Theorem 3. Suppose that $w \geq 0, \mathfrak{R}(p) \geq 0, \rho$ and $\delta \in \mathbb{C}$. Then, the following Whittaker transform formula holds:

$$
\begin{aligned}
\int_{0}^{\infty} & t^{\rho-1} e^{-\delta t / 2} W_{\lambda, \mu}(\delta t) F_{p}^{(\alpha, \beta ; m)}(a, b ; c ; w t) d t \\
= & \delta^{-\rho} \frac{\Gamma((1 / 2)+\mu+\rho) \Gamma((1 / 2)-\mu+\rho)}{\Gamma(1-\lambda+\rho)} \\
& \quad \times{ }_{2} F_{p, 1}^{(\alpha, \beta ; m)}\left(a, \frac{1}{2}+\mu+\rho, \frac{1}{2}-\mu+\rho, b ; c, 1-\lambda+\rho ; \frac{w}{\delta}\right),
\end{aligned}
$$

provided that the integral involving Whittaker transform $F_{p}^{(\alpha, \beta ; m)}$ converges.

Proof. Applying (8) and setting $\delta t=v$ in the left-hand side of (20), we get

$$
\begin{aligned}
& \int_{0}^{\infty}\left(\frac{\nu}{\delta}\right)^{\rho-1} e^{-\nu / 2} W_{\lambda, \mu}(\nu) \\
& \quad \times \sum_{n=0}^{\infty}(a)_{n} \frac{B_{p}^{(\alpha, \beta ; m)}(b+n, c-b)}{B(b, c-b)} \frac{(w \nu)^{n}}{\delta^{n} \delta n !} d \nu .
\end{aligned}
$$

By changing the order of integration and summation, we obtain

$$
\begin{gathered}
\delta^{-\rho} \sum_{n=0}^{\infty}(a)_{n} \frac{B_{p}^{(\alpha, \beta ; m)}(b+n, c-b)}{B(b, c-b)} \frac{w^{n}}{\delta^{n} n !} \\
\times \int_{0}^{\infty} \nu^{\rho+n-1} e^{-\nu / 2} W_{\lambda, \mu}(\nu) d \nu .
\end{gathered}
$$

Next, we can use the following integral formula involving the Whittaker function:

$$
\begin{aligned}
& \int_{0}^{\infty} t^{\nu-1} e^{-t / 2} W_{\lambda, \mu}(t) d t \\
& =\frac{\Gamma((1 / 2)+\mu+\nu) \Gamma((1 / 2)-\mu+\nu)}{\Gamma((1 / 2)-\lambda+\nu)} \quad\left(\Re(\nu \pm \mu)>-\frac{1}{2}\right) .
\end{aligned}
$$

Then, after a little simplification, (22) becomes the following form:

$$
\begin{aligned}
\int_{0}^{\infty} t^{\rho-1} e^{-\delta t / 2} W_{\lambda, \mu}(\delta t) F_{p}^{(\alpha, \beta ; m)}(a, b ; c ; w t) d t \\
=\delta^{-\rho} \sum_{n=0}^{\infty}(a)_{n} \frac{B_{p}^{(\alpha, \beta ; m)}(b+n, c-b)}{B(b, c-b)} \frac{w^{n}}{\delta^{n} n !} \\
\quad \times \frac{\Gamma((1 / 2)+\mu+\rho+n) \Gamma((1 / 2)-\mu+\rho+n)}{\Gamma(1-\lambda+\rho+n)},
\end{aligned}
$$

which, upon using (8), yields our desired result (20).

It may be noted in passing that the special cases of Theorems 1 to 3 when $m=1$ immediately reduce to the corresponding results due to Agarwal [11]. 


\section{Fractional Calculus of the Generalized Gauss Hypergeometric Functions}

Recently, fractional integral operators involving the various special functions have been investigated by several authors (see, e.g., [11, 13-26]; see also [27, 28]). Here, in this section, we will establish some fractional integral formulas for the generalized Gauss hypergeometric type functions $F_{p}^{(\alpha, \beta ; m)}(a, b ; c ; z)$. To do this, we need to recall the following pair of Saigo hypergeometric fractional integral operators.

For $x>0, \mu, \nu, \eta \in \mathbb{C}$ and $\mathfrak{R}(\alpha)>0$, we have

$$
\begin{aligned}
\left(I_{0, x}^{\mu, \nu, \eta} f(t)\right)(x)= & \frac{x^{-\mu-\nu}}{\Gamma(\mu)} \int_{0}^{x}(x-t)^{\mu-1} \\
& \times{ }_{2} F_{1}\left(\mu+\nu,-\eta ; \mu ; 1-\frac{t}{x}\right) f(t) d t \\
\left(J_{x, \infty}^{\mu, \nu, \eta} f(t)\right)(x)= & \frac{1}{\Gamma(\mu)} \int_{x}^{\infty}(t-x)^{\mu-1} t^{-\mu-\nu} \\
& \times{ }_{2} F_{1}\left(\mu+\nu,-\eta ; \mu ; 1-\frac{x}{t}\right) f(t) d t
\end{aligned}
$$

where the ${ }_{2} F_{1}(\cdot)$, a special case of the generalized hypergeometric function (10), is the Gauss hypergeometric function.

The operator $I_{0, x}^{\mu, \nu, \eta}(\cdot)$ contains both the RiemannLiouville $R_{0, x}^{\mu}(\cdot)$ and the Erdélyi-Kober $E_{0, x}^{\mu, \eta}(\cdot)$ fractional integral operators by means of the following relationships:

$$
\begin{aligned}
\left(R_{0, x}^{\mu} f(t)\right)(x) & =\left(I_{0, x}^{\mu,-\mu, \eta} f(t)\right)(x) \\
& =\frac{1}{\Gamma(\mu)} \int_{0}^{x}(x-t)^{\mu-1} f(t) d t, \\
\left(E_{0, x}^{\mu, \eta} f(t)\right)(x) & =\left(I_{0, x}^{\mu, 0, \eta} f(t)\right)(x) \\
& =\frac{x^{-\mu-\eta}}{\Gamma(\mu)} \int_{0}^{x}(x-t)^{\mu-1} t^{\eta} f(t) d t .
\end{aligned}
$$

It is noted that the operator (26) unifies the Weyl type and the Erdélyi-Kober fractional integral operators as follows:

$$
\begin{aligned}
\left(W_{x, \infty}^{\mu} f(t)\right)(x) & =\left(J_{x, \infty}^{\mu,-\mu, \eta} f(t)\right)(x) \\
& =\frac{1}{\Gamma(\mu)} \int_{x}^{\infty}(t-x)^{\mu-1} f(t) d t \\
\left(K_{x, \infty}^{\mu, \eta} f(t)\right)(x) & =\left(J_{x, \infty}^{\mu, 0, \eta} f(t)\right)(x) \\
& =\frac{x^{\eta}}{\Gamma(\mu)} \int_{x}^{\infty}(t-x)^{\mu-1} t^{-\mu-\eta} f(t) d t
\end{aligned}
$$

We also use the following image formulas which are easy consequences of the operators (25) and (26) (see $[24,26])$ :

$$
\begin{array}{r}
\left(I_{0, x}^{\mu, \nu, \eta} t^{\lambda-1}\right)(x)=\frac{\Gamma(\lambda) \Gamma(\lambda-\nu+\eta)}{\Gamma(\lambda-\nu) \Gamma(\lambda+\mu+\eta)} x^{\lambda-\nu-1} \\
(\lambda>0, \lambda-\nu+\eta>0), \\
\left(J_{x, \infty}^{\mu, \nu, \eta} t^{\lambda-1}\right)(x)=\frac{\Gamma(\nu-\lambda+1) \Gamma(\eta-\lambda+1)}{\Gamma(1-\lambda) \Gamma(\nu+\mu-\lambda+\eta+1)} x^{\lambda-\nu-1} \\
\quad(\beta-\lambda+1>0, \eta-\lambda+1>0) .
\end{array}
$$

The Saigo fractional integrations of generalized Gauss hypergeometric type functions (8) are given by the following results.

Theorem 4. Let $x>0, \mathfrak{R}(p) \geq 0$, and $\mu, \nu, \eta, \rho, e \in \mathbb{C}$ be parameters such that

$$
\mathfrak{R}(\mu)>0, \quad \mathfrak{R}(\rho)>0, \quad \mathfrak{R}(\rho)>\max \{0, \mathfrak{R}(\nu-\eta)\} .
$$

Then, the following fractional integral formula holds:

$$
\begin{aligned}
& \left(I_{0, x}^{\mu, \nu, \eta}\left[t^{\rho-1} F_{p}^{(\alpha, \beta ; m)}(a, b ; c ; e t)\right]\right)(x) \\
& =x^{\rho-\nu-1} \frac{\Gamma(\rho) \Gamma(\rho-\nu+\eta)}{\Gamma(\rho+\mu+\eta) \Gamma(\rho-\nu)} \\
& \quad \times{ }_{2} F_{p, 2}^{(\alpha, \beta ; m)}\left[\begin{array}{lr}
a, b, \rho, \rho-\nu+\eta ; & \\
c, \rho-\nu, \rho+\mu+\eta ; & e x
\end{array}\right] .
\end{aligned}
$$

Proof. For convenience, we denote the left-hand side of the result (34) by $\mathscr{I}$. Using (8), and then changing the order of integration and summation, which is valid under the conditions of Theorem 4 , we find

$$
\begin{aligned}
\mathscr{I} & =\left(I_{0, t}^{\mu, v, \eta}\left[t^{\rho-1} \sum_{n=0}^{\infty} a_{n} \frac{B_{p}^{(\alpha, \beta ; m)}(b+n, c-b)}{B(b, c-b)} \frac{(e t)^{n}}{n !}\right]\right)(x) \\
& =\sum_{n=0}^{\infty} a_{n} \frac{B_{p}^{(\alpha, \beta ; m)}(b+n, c-b)}{B(b, c-b)} \frac{e^{n}}{n !}\left(I_{0, t}^{\mu, v, \eta}\left\{t^{\rho+n-1}\right\}\right)(x) .
\end{aligned}
$$

Now, making use of the result (31), we obtain

$$
\begin{aligned}
\mathscr{I}= & x^{\rho-\nu-1} \sum_{n=0}^{\infty} a_{n} \frac{B_{p}^{(\alpha, \beta ; m)}(b+n, c-b)}{B(b, c-b)} \\
& \times \frac{\Gamma(\rho+n) \Gamma(\rho-\nu+\eta+n)}{\Gamma(\rho-\nu+n) \Gamma(\rho+\mu+\eta+n)} \frac{(e x)^{n}}{n !} .
\end{aligned}
$$

This, in view of (8), gives the desired result (34). 
Theorem 5. Let $x>0, \mathfrak{R}(p) \geq 0$, and $\mu, v, \eta, \rho, e \in \mathbb{C}$ be parameters satisfying the following inequalities:

$$
\mathfrak{R}(\mu)>0, \quad \mathfrak{R}(\rho)>0, \quad \mathfrak{R}(\rho)<1+\min \{\mathfrak{R}(\eta), \mathfrak{R}(\nu)\} .
$$

Then, the following fractional integral formula holds:

$$
\begin{aligned}
& \left(J_{x, \infty}^{\mu, \nu, \eta}\left[t^{\rho-1} F_{p}^{(\alpha, \beta ; m)}\left(a, b ; c ; \frac{e}{t}\right)\right]\right)(x) \\
& =x^{\rho-\nu-1} \frac{\Gamma(1-\rho+\nu) \Gamma(1-\rho+\eta)}{\Gamma(1-\rho) \Gamma(1-\rho-\eta+\nu+\mu)} \\
& \quad \times{ }_{2} F_{p, 2}^{(\alpha, \beta ; m)}\left[\begin{array}{ll}
a, b, 1-\rho+\nu, 1-\rho+\eta ; & e \\
c, 1-\rho, 1-\rho+\mu+\nu-\eta ; & \frac{e}{x}
\end{array}\right] .
\end{aligned}
$$

Proof. As in the proof of Theorem 4, taking the operator (26) and the result (32) into account, one can easily prove (38). Therefore, we omit the details of the proof.

Setting $v=0$ in Theorems 4 and 5 and employing the relations (28) and (30) yield certain interesting results asserted by the following corollaries.

Corollary 6. Let $x>0, \mathfrak{R}(p) \geq 0$, and $\mu, \eta, \rho, e \in \mathbb{C}$ be parameters such that $\mathfrak{R}(\mu)>0, \mathfrak{R}(\rho)>0$, and $\mathfrak{R}(\rho)>$ $\mathfrak{R}(-\eta)$. Then, the right-side Erdélyi-Kober fractional integrals of the generalized Gauss hypergeometric type functions are given by

$$
\begin{aligned}
& \left(E_{0, x}^{\mu, \eta}\left[t^{\rho-1} F_{p}^{(\alpha, \beta ; m)}(a, b ; c ; e t)\right]\right)(x) \\
& =x^{\rho-1} \frac{\Gamma(\rho-\eta)}{\Gamma(\rho+\mu+\eta)} \\
& \quad \times{ }_{1} F_{p, 1}^{(\alpha, \beta ; m)}\left[\begin{array}{ll}
a, b, \rho-\eta ; & \\
c, \rho+\mu+\eta ; & e x
\end{array}\right] .
\end{aligned}
$$

Corollary 7. Let $x>0, \mathfrak{R}(p) \geq 0$, and $\mu, \eta, \rho, e \in \mathbb{C}$ be parameters satisfying the following inequalities $\mathfrak{R}(\mu)>0$, $\mathfrak{R}(\rho)>0$, and $\mathfrak{R}(\rho)<1+\mathfrak{R}(\eta)$. Then, one has

$$
\begin{aligned}
& \left(K_{x, \infty}^{\mu, \eta}\left[t^{\rho-1} F_{p}^{(\alpha, \beta ; m)}\left(a, b ; c ; \frac{e}{t}\right)\right]\right)(x) \\
& =x^{\rho-1} \frac{\Gamma(1-\rho+\eta)}{\Gamma(1-\rho-\eta+\mu)} \\
& \quad \times{ }_{1} F_{p, 1}^{(\alpha, \beta ; m)}\left[\begin{array}{cc}
a, b, 1-\rho+\eta ; & e \\
c, 1-\rho+\mu-\eta ; & \frac{e}{x}
\end{array}\right] .
\end{aligned}
$$

Further, if we replace $\nu$ with $-\mu$ in Theorems 4 and 5 and make use of the relations (27) and (29), we obtain other Riemann-Liouville and Weyl fractional integrals of the generalized Gauss hypergeometric type function $F_{p}^{(\alpha, \beta ; m)}$ given by the following corollaries.
Corollary 8. Let $x>0, \mathfrak{R}(p) \geq 0$, and $\mu, \rho \in \mathbb{C}$ be parameters such that $\mathfrak{R}(\mu)>0$ and $\mathfrak{R}(\rho)>0$. Then, one gets

$$
\begin{aligned}
& \left(R_{0, x}^{\mu}\left[t^{\rho-1} F_{p}^{(\alpha, \beta ; m)}(a, b ; c ; e t)\right]\right)(x) \\
& =x^{\rho+\mu-1} \frac{\Gamma(\rho)}{\Gamma(\rho+\mu)}{ }_{1} F_{p, 1}^{(\alpha, \beta ; m)}\left[\begin{array}{cc}
a, b, \rho ; & \\
c, \rho+\mu ; & e x
\end{array}\right] .
\end{aligned}
$$

Corollary 9. Let $x>0, \mathfrak{R}(p) \geq 0$, and $\mu, \rho \in \mathbb{C}$ be parameters satisfying the following inequalities $\mathfrak{R}(\mu)>0$ and $\mathfrak{R}(\rho)>0$. Then, one obtains

$$
\begin{aligned}
& \left(W_{x, \infty}^{\mu}\left[t^{\rho-1} F_{p}^{(\alpha, \beta ; m)}\left(a, b ; c ; \frac{e}{t}\right)\right]\right)(x) \\
& =x^{\rho+\mu-1} \frac{\Gamma(1-\rho-\mu)}{\Gamma(1-\rho)}{ }_{1} F_{p, 1}^{(\alpha, \beta ; m)}\left[\begin{array}{cc}
a, b, 1-\rho-\mu ; & \frac{e}{x} \\
c, 1-\rho ; &
\end{array} .\right.
\end{aligned}
$$

\section{Concluding Remarks}

We can also present a large number of special cases of our main fractional integral formulas in Theorems 4 and 5. Here, we illustrate two more formulas. Setting $\alpha=\beta$ in (34) and (38), respectively, and using the known formula due to Lee et al. (see, [5, page 197, Equation (6.1)]), we obtain certain interesting (presumably) new fractional integral formulas involving the extended hypergeometric function $F_{p}^{(m)}(a, b ; c ; z)$ asserted by the following corollaries.

Corollary 10. Let $x>0, \mathfrak{R}(p) \geq 0$, and $\mu, \nu, \eta, \rho, e \in \mathbb{C}$ be parameters such that

$$
\mathfrak{R}(\mu)>0, \quad \mathfrak{R}(\rho)>0, \quad \mathfrak{R}(\rho)>\max \{0, \mathfrak{R}(\nu-\eta)\} .
$$

Then, one has

$$
\begin{aligned}
& \left(I_{0, x}^{\mu, \nu, \eta}\left[t^{\rho-1} F_{p}^{m}(a, b ; c ; e t)\right]\right)(x) \\
& =x^{\rho-\nu-1} \frac{\Gamma(\rho) \Gamma(\rho-\nu+\eta)}{\Gamma(\rho+\mu+\eta) \Gamma(\rho-\nu)} \\
& \quad \times{ }_{2} F_{p, 2}^{m}\left[\begin{array}{ll}
a, b, \rho, \rho-\nu+\eta ; & \\
c, \rho-\nu, \rho+\mu+\eta ; & e x
\end{array}\right] .
\end{aligned}
$$

Corollary 11. Let $x>0, \mathfrak{R}(p) \geq 0$, and $\mu, \nu, \eta, e, \rho \in \mathbb{C}$ be parameters satisfying the following inequalities:

$\mathfrak{R}(\mu)>0, \quad \mathfrak{R}(\rho)>0, \quad \mathfrak{R}(\rho)<1+\min \{\mathfrak{R}(\eta), \mathfrak{R}(\nu)\}$. 
Then, one gets

$$
\begin{aligned}
& \left(J_{x, \infty}^{\mu, \nu, \eta}\left[t^{\rho-1} F_{p}\left(a, b ; c ; \frac{e}{t}\right)\right]\right)(x) \\
& =x^{\rho-\beta-1} \frac{\Gamma(1-\rho+\nu) \Gamma(1-\rho+\eta)}{\Gamma(1-\rho) \Gamma(1-\rho-\eta+\nu+\mu)} \\
& \quad \times{ }_{2} F_{p, 2}\left[\begin{array}{ll}
a, b, 1-\rho+\nu, 1-\rho+\eta ; & \frac{e}{x} \\
c, 1-\rho, 1-\rho+\mu+\nu-\eta ;
\end{array}\right] .
\end{aligned}
$$

Setting $m=1$ and $\alpha=\beta$ in Theorems 4 and 5 and making use of the relation (6) yield some known fractional integral formulas due to Agarwal [11]. Also setting $m=1$ and making use of the relation (7) give the known integral transforms and fractional integral formulas due to Agarwal [11]. Further, if we set $m=1$ and $p=0$ in Theorems 1 to 5 or make use of the result (8), we obtain various integral transforms and fractional integral formulas for the Gauss hypergeometric function ${ }_{2} F_{1}$.

The generalized Gauss hypergeometric type functions defined by (8) possess the advantage that most of the known and widely investigated special functions are expressible in terms of the generalized Gauss hypergeometric functions $F_{p}^{(\alpha, \beta ; m)}$ (see [9]). We may also emphasize that results derived in this paper are of general character and can specialize to give further interesting and potentially useful formulas involving integral transform and fractional calculus. Finally, it is expected that the results presented here with potential special cases can find some applications in probability theory and to the solutions of fractional differential and integral equations.

\section{Conflict of Interests}

The authors declare that there is no conflict of interests regarding the publication of this paper.

\section{Acknowledgments}

The authors would like to express their deep gratitude for the reviewers' helpful comments. This research was, in part, supported by the Basic Science Research Program through the National Research Foundation of Korea funded by the Ministry of Education, Science and Technology of the Republic of Korea (Grant no. 2010-0011005). This work was supported by Dongguk University Research Fund.

\section{References}

[1] H. M. Srivastava and J. Choi, Zeta and q-Zeta Functions and Associated Series and Integrals, Elsevier Science, Amsterdam, The Netherlands, 2012.

[2] P. Agarwal, M. Chand, and S. D. Purohit, "A note on generating functions involving generalized Gauss hypergeometric functions," National Academy Science Letters. In press.

[3] M. A. Chaudhry, A. Qadir, M. Rafique, and S. M. Zubair, "Extension of Euler's beta function," Journal of Computational and Applied Mathematics, vol. 78, no. 1, pp. 19-32, 1997.
[4] M. A. Chaudhry, A. Qadir, H. M. Srivastava, and R. B. Paris, "Extended hypergeometric and confluent hypergeometric functions," Applied Mathematics and Computation, vol. 159, no. 2, pp. 589-602, 2004.

[5] D. M. Lee, A. K. Rathie, R. K. Parmar, and Y. S. Kim, "Generalization of extended beta function, hypergeometric and confluent hypergeometric functions," Honam Mathematical Journal, vol. 33, no. 2, pp. 187-206, 2011.

[6] E. Özergin, Some properties of hypergeometric functions [Ph.D. thesis], Eastern Mediterranean University, Gazimağusa, North Cyprus, 2011.

[7] E. Özergin, M. A. Özarslan, and A. Altın, "Extension of gamma, beta and hypergeometric functions," Journal of Computational and Applied Mathematics, vol. 235, no. 16, pp. 4601-4610, 2011.

[8] H. Liu and W. Wang, "Some generating relations for extended Appell's and Lauricella's hypergeometric functions," The Rocky Mountain Journal of Mathematics. In press.

[9] R. K. Parmar, "A new generalization of gamma, beta, hypergeometric and confluent hypergeometric functions," Le Matematiche, vol. 68, no. 2, pp. 33-42, 2013.

[10] H. M. Srivastava and P. W. Karlsson, Multiple Gaussian Hypergeometric Series, Halsted Press, Ellis Horwood Limited, Chichester, UK, John Wiley and Sons, New York, NY, USA, 1985.

[11] P. Agarwal, "Certain properties of the generalized Gauss hypergeometric functions," Applied Mathematics \& Information Sciences, vol. 8, no. 5, pp. 2315-2320, 2014.

[12] I. N. Sneddon, The Use of Integral Transforms, Tata McGrawHill, New Delhi, India, 1979.

[13] P. Agarwal, "Fractional integration of the product of two multivariables $H$-function and a general class of polynomials," in Advances in Applied Mathematics and Approximation Theory, vol. 41 of Springer Proceedings in Mathematics \& Statistics, pp. 359-374, Springer, New York, NY, USA, 2013.

[14] P. Agarwal, "Further results on fractional calculus of Saigo operators," Applications and Applied Mathematics, vol. 7, no. 2, pp. 585-594, 2012.

[15] P. Agarwal, "Generalized fractional integration of the $\bar{H}$ function," Le Matematiche, vol. 67, no. 2, pp. 107-118, 2012.

[16] P. Agarwal and S. Jain, "Further results on fractional calculus of Srivastava polynomials," Bulletin of Mathematical Analysis and Applications, vol. 3, no. 2, pp. 167-174, 2011.

[17] P. Agarwal and S. D. Purohit, "The unified pathway fractional integral formulae," Journal of Fractional Calculus and Applications, vol. 4, no. 9, pp. 1-8, 2013.

[18] A. A. Kilbas, "Fractional calculus of the generalized Wright function," Fractional Calculus \& Applied Analysis, vol. 8, no. 2, pp. 113-126, 2005.

[19] A. A. Kilbas, H. M. Srivastava, and J. J. Trujillo, Theory and Applications of Fractional Differential Equations, vol. 204 of North-Holland Mathematical Studies, Elsevier Science, Amsterdam, The Netherlands, 2006.

[20] V. Kiryakova, "On two Saigo's fractional integral operators in the class of univalent functions," Fractional Calculus \& Applied Analysis, vol. 9, no. 2, pp. 160-176, 2006.

[21] V. Kiryakova, "A brief story about the operators of the generalized fractional calculus," Fractional Calculus \& Applied Analysis, vol. 11, no. 2, pp. 203-220, 2008.

[22] S. Kumar, D. Kumar, S. Abbasbandy, and M. M. Rashidi, "Analytical solution of fractional Navier-Stokes equation byusing modifed Laplace decomposition method," Ain Shams Engineering Journal, vol. 5, pp. 569-574, 2014. 
[23] M. Saigo, "On generalized fractional calculus operators," in Proceedings of the International Workshop on Recent Advances in Applied Mathematics, pp. 441-4450, Kuwait University, Kuwait, 1996.

[24] M. Saigo, "A remark on integral operators involving the Gauss hypergeometric functions," Mathematical Reports of College of General Education. Kyushu University, vol. 11, no. 2, pp. 135-143, 1978.

[25] M. Saigo, "A certain boundary value problem for the EulerDarboux equation I," Mathematica Japonica, vol. 24, no. 4, pp. 377-385, 1979.

[26] M. Saigo and N. Maeda, "More generalization of fractional calculus," in Proceedings of the 2nd International Workshop on Transform Metods and Special Functions, P. Rusev, I. Dimovski, and V. Kiryakova, Eds., pp. 386-400, IMI, Varna, Bulgaria, August 1996.

[27] H. M. Srivastava and P. Agarwal, "Certain fractional integral operators and the generalized incomplete hypergeometric functions," Applications and Applied Mathematics, vol. 8, no. 2, pp. 333-345, 2013.

[28] A.-M. Yang, Y.-Z. Zhang, C. Cattani et al., "Application of local fractional series expansion method to solve Klein-Gordon equations on Cantor sets," Abstract and Applied Analysis, vol. 2014, Article ID 372741, 6 pages, 2014. 


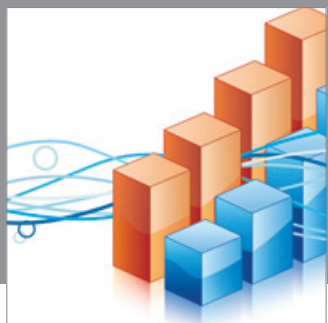

Advances in

Operations Research

mansans

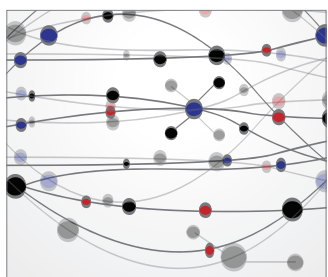

The Scientific World Journal
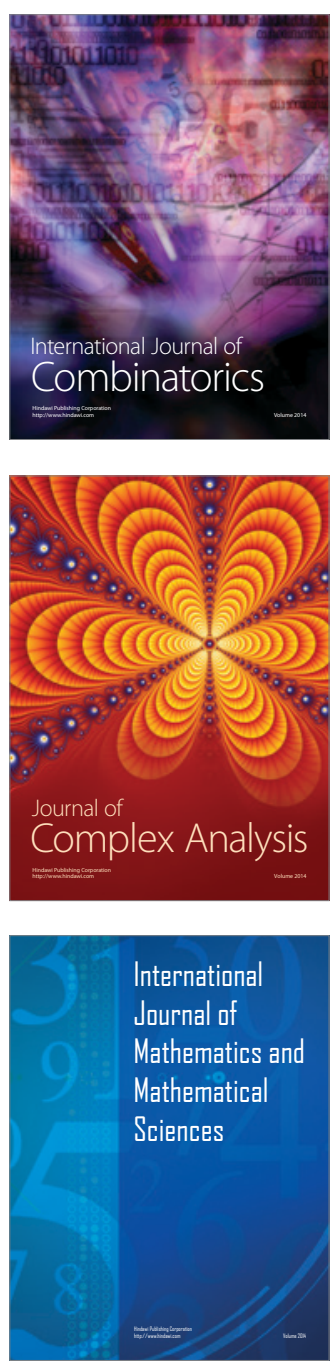
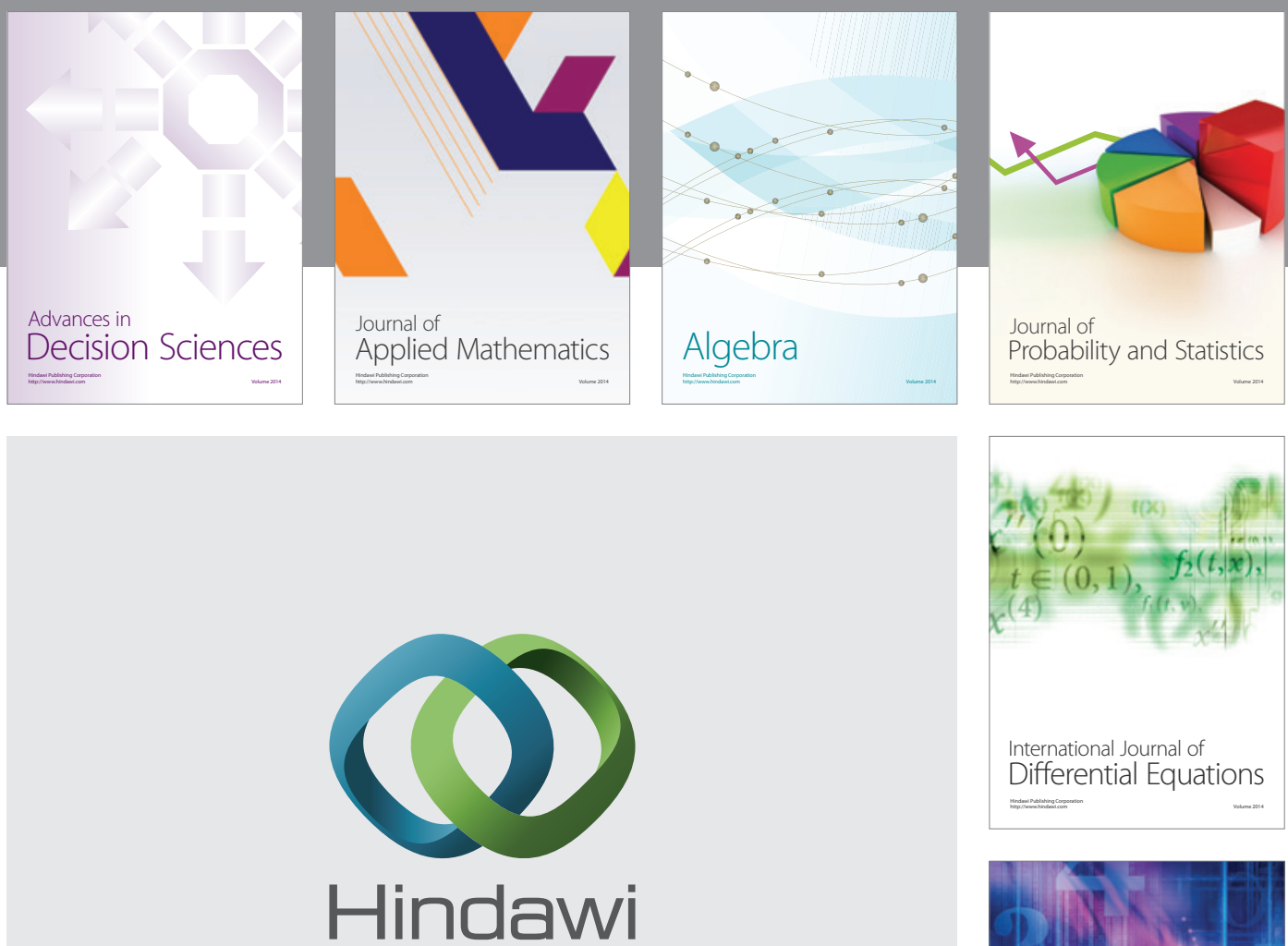

Submit your manuscripts at http://www.hindawi.com
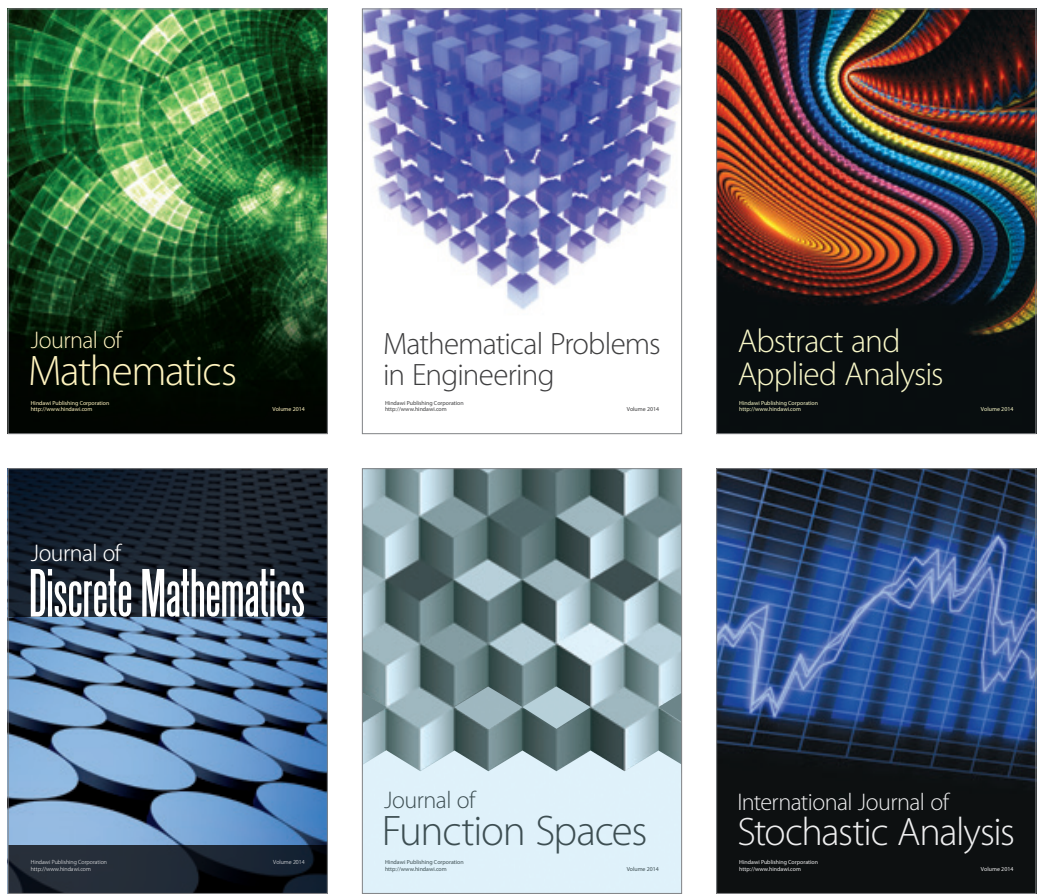

Journal of

Function Spaces

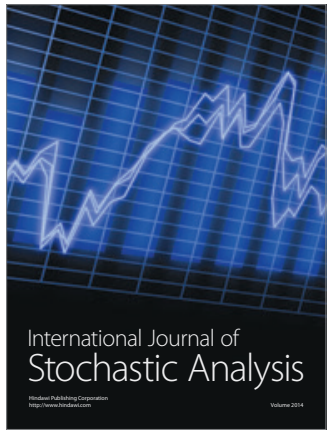

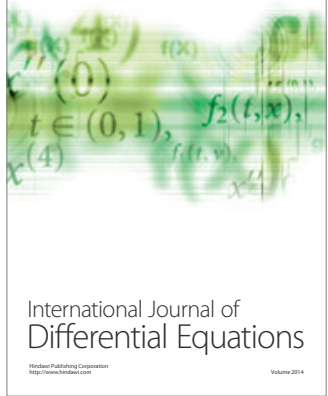
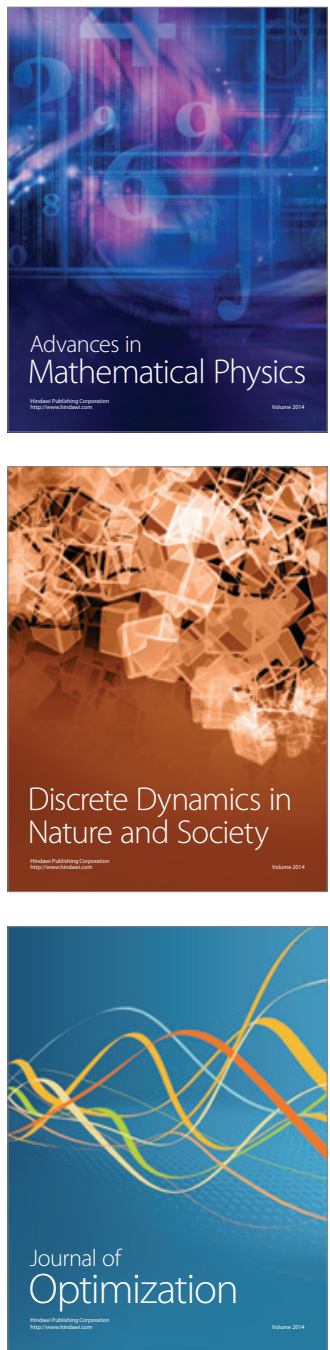